\title{
El Liderazgo para la Mejora Escolar y la Justicia Social. Un Estudio de Caso sobre un Centro de Educación Secundaria Obligatoria
}

\author{
The Leadership for School Improvement and Social Justice. \\ A case Study in Secondary Education School
}

\author{
Estefanía Martínez-Valdivia ${ }^{1 *}$ \\ Inmaculada García-Martínez ${ }^{2}$ \\ María Lina Higueras-Rodríguez ${ }^{2}$ \\ ${ }^{1}$ Universidad de Jaén, España \\ ${ }^{2}$ Universidad de Granada, España
}

\begin{abstract}
Esta investigación es un estudio de caso de un centro de Educación Secundaria Obligatoria, se persigue el mismo objetivo que el proyecto del que forma parte, que es: conocer las prácticas exitosas de liderazgo para la mejora de los resultados académicos. Se ha utilizado el estudio de caso como metodología y la entrevista y la revisión de documentos como instrumentos de investigación. Los resultados obtenidos resaltan las características y estrategias exitosas de liderazgo para conseguir buenos resultados académicos aun siendo un centro con unas características no demasiado favorecedoras. Los resultados indican que el director desarrolla un liderazgo distribuido y participativo, subrayando las características relacionadas con su carácter, formación, capacidad de mejora, de conseguir desarrollar un ambiente favorable hacia el trabajo tanto para el alumnado como el profesorado, el compartir una visión clara y para ello trabajar de un modo conjunto con la comunidad educativa (alumnado, profesorado, familias, etc.). Las conclusiones obtenidas están relacionadas con los resultados del proyecto ISSPP, relativas a: la personalización de la experiencia de aprendizaje, el trabajo colaborativo de los docentes, la retroalimentación de la comunidad educativa para la mejora de la escuela, entre otras.
\end{abstract}

Descriptores: Liderazgo; Director; Mejora; Justicia Social; Escuela.

This research is a case study of a compulsory secondary education center, the same objective is pursued as the project of which it is part that is: to know the successful practices of leadership for the improvement of academic results. The case study has been used as a methodology and the interview and the review of documents as research instruments. The results obtained are focused on highlighting the characteristics and successful leadership strategies to achieve good academic results. The results indicate that the director develops a distributed and participative leadership, underlining the characteristics related to his character, training, capacity for improvement, of developing a favorable environment towards work both for students and teachers, to share a clear vision and to do this, work in a joint way with the educational community (students, teachers, families, etc.). The conclusions obtained are related to the results of the ISSPP project, related to: the personalization of the learning experience, the collaborative work of the teachers, the feedback of the educational community for the improvement of the school, among others.

Keywords: Leadership; School principal; Improvement; Social justice; School.

Investigación que forma parte del Proyecto Internacional Succesful School Principaship Project (ISSPP) dirigido por Cristopher Day de la Universidad de Nottinghan.

*Contacto: evaldivi@ujaen.es

ISSN: 1696-4713

www.rinace.net/reice/

revistas.uam.es/reice
Recibido: $\quad 5$ de septiembre 2017

$1^{\text {a }}$ Evaluación: 23 de octubre 2017

$2^{\text {a }}$ Evaluación: 10 de noviembre 2017

Aceptado: $\quad 14$ de diciembre 2017 


\section{Introducción}

La investigación que presentamos se encuadra dentro del Proyecto International denominado Succesful School Principaship Project (ISSPP). Se inició en 2001 en Nottinghan (Inglaterra) bajo la dirección de Chistopher Day. Es un proyecto muy ambicioso en el que pronto se han ido sumando países de todo el mundo, estando en la actualidad trabajando en el mismo académico de 24 países.

Este proyecto gira en torno a una pregunta general ¿Qué actuaciones de liderazgo exitoso existen en las escuelas de todo el mundo? Y en la misma se basan estos objetivos: 1) Identificar los valores, conocimientos y habilidades que los líderes escolares exitosos usan para efectuar las prácticas de liderazgo en escuelas exitosas de distintos países; 2) Identificar las prácticas de liderazgo eficaces en las escuelas y 3) Conocer la relación entre las prácticas exitosas y los resultados escolares de los diferentes países (Day, 2015). Con este proyecto se pretende que a través de estudios de caso de escuelas de todo el mundo se obtenga la primera base de datos internacional sobre liderazgo escolar exitoso sostenible. En la actualidad ya se han publicado numerosos artículos en las mejores revistas de impacto, así como libros en editoriales de renombre con resultados obtenidos en torno a este proyecto.

Entre los países participantes en el mismo se encuentra España, que colabora a través de la Red de excelencia RILME (Red de Investigación sobre Liderazgo y Mejora Educativa) formada por equipos de investigación pertenecientes a diferentes universidades españolas. Con esta investigación aportamos un estudio de caso vinculado con el proyecto ISSPP. Contextualizándolo, es un centro de Educación Secundaria Obligatoria ubicado en un contex to desfavorable donde existe una gran diversidad de alumnado, pero que pese a sus características obtiene buenos resultados académicos.

La metodología utilizada es la misma que se lleva a cabo en el Proyecto ISSPP, que a través del estudio de casos pretende: recoger datos desde diferentes perspectivas (directores, padres, madres, estudiantes y profesores) e identificar las cualidades personales y competencias profesionales genéricas de los líderes escolares eficaces (Day et al., 2000). También los instrumentos de investigación utilizados son los ofrecidos por el proyecto, pues ello permite conocer las prácticas exitosas de liderazgo para la mejora de los resultados académicos que persigue este estudio mediante un caso relevante para el mismo.

\section{Liderazgo educativo para la mejora escolar}

El liderazgo escolar es un tema de gran actualidad entre los académicos del ámbito de la educación. Pues cuando se trata de reformas del sistema educativo a nivel internacional, el rol del director es una gran meta para la reforma y el vehículo para conseguir otros cambios significativos (Leithwood y Riehl, 2009). Autores como Hallinger y Heck (2014) resaltan que el liderazgo contribuye el aprendizaje a través de la formación de una serie de procesos estructurales y también socioculturales que definen la capacidad de la escuela para la mejora escolar.

Cuando hablamos de reforma escolar autores como Hopkins (2013) señala la importancia de conseguir que el foco de la misma sea el estudiante y su aprendizaje. En su trabajo indica diez claves para la reforma de un sistema educativo, estando entre ellas la relevancia 
de un liderazgo centrado en los procesos de aprendizaje. También debe promoverse el liderazgo distribuido pues para mejorar la enseñanza en el aula es necesario apoyar, estimular e influir en el profesorado. Como indican Bolívar (2014) y Day y sus colaboradores (2011), cuando la dirección escolar es entendida como liderazgo educativo tiene una responsabilidad eje en sus actuaciones que consiste en conseguir la mejora del aprendizaje de los estudiantes, pero también lograr ayudar a los profesores para este fin.

El liderazgo habitual con el que estamos familiarizados en las instituciones educativas españolas, concretamente, está relacionado con funciones como la gestión principalmente, siendo un liderazgo tan individualizado y coartado por la burocracia administrativa, que en la actualidad queda en entre dicho (Bolívar, 2010). Por ello para conseguir una escuela con capacidad de mejora se apuesta por un liderazgo educativo y distribuido, llevando a cabo funciones que conduzcan a una construcción social, sobrepasando únicamente la gestión del centro (Hopkins, Ainscow y West, 1994; Stoll y Fink, 1998).

Tales líderes desarrollan determinadas actuaciones clave que les diferencian, llevándoles a realizar un liderazgo exitoso, el cuadro 1 representa algunas de las más relevantes.

La investigación sobre el liderazgo avanza entorno la necesidad de mejorar y profundizar en sus funciones para conseguir la capacidad de mejora de la escuela, siendo el centro de interés el estudiante. Pues como vemos, conseguir hacer realidad este tipo de liderazgo no es algo fácil, pues estamos habituados a que éste sea individualista y jerárquico considerándose el menos idóneo para impulsar la capacidad de mejora en los centros.

Por este motivo la investigación que presentamos se centra en la línea uno y dos del proyecto ISSPP que persigue el siguiente objetivo: conocer las prácticas exitosas de liderazgo para la mejora de los resultados académicos desde diferentes perspectivas.

\section{Método}

La investigación que presentamos es de corte cualitativo. El método de investigación utilizado y los instrumentos seleccionados están marcados por el proyecto ISSPP en el que se encuadra este trabajo. Se ha utilizado el estudio de casos como la metodología más idónea para comprender la realidad del liderazgo escolar en el centro objeto de estudio. Como instrumentos de investigación se realizaron entrevistas en profundidad al director, profesores, alumnado, padres y madres, y para completar y contrastar resultados se llevó a cabo revisión de documentos.

El caso que presentamos se seleccionó intencionalmente, teniendo que cumplir algunas características concretas como las siguientes: que estuviera ubicado en un contexto desafiante, donde exista una gran diversidad cultural con riesgo de exclusión social y educativa. Pero siendo, además, un centro caracterizado por su elevado rendimiento académico y sus adecuadas actuaciones para conseguir la mejora escolar. Para comprobar tales características del centro, revisamos los resultados académicos del curso escolar 2013/2014 que muestran los indicadores homologados para la autoevaluación de centros que imparten Educación Secundaria (AGAVE, 2014). 
Cuadro 1. Claves del liderazgo exitoso para la mejora escolar.

\begin{tabular}{|c|c|}
\hline INVESTIGACIONES & CARACTERÍSTICAS DE LIDERAZGO EXITOSO \\
\hline $\begin{array}{l}\text { Leithwood y Riehl } \\
\text { (2009) }\end{array}$ & $\begin{array}{l}\text { Liderazgo para la mejora escolar } \\
\text { Liderazgo ejercido por los directores, por los profesores y también puede } \\
\text { ser distribuido a otros. } \\
\text { Un conjunto de prácticas básicas puede ser exitosas en cualquier contexto: } \\
\text { - Establecer rumbos: identificar y articular una visión, fomentar la } \\
\text { aceptación de metas grupales, generar altas expectativas de } \\
\text { rendimiento. } \\
\text { - Desarrollar a las personas: ofrecer estímulo intelectual, } \\
\text { proporcionar apoyo individualizado, proveer un modelo adecuado. } \\
\text { - Rediseño de la organización: fortalecer la cultura escolar, modificar } \\
\text { estructuras organizacionales, construir procesos colaborativos. } \\
\text { - Los líderes que trabajan con diferentes tipos de alumnos establecen } \\
\text { las condiciones idóneas para promover el logro escolar, la equidad } \\
\text { y la justicia: } \\
\text { - Construir formas potentes de enseñanza y aprendizaje. } \\
\text { - Crear comunidades estables en la escuela. } \\
\text { - Promover el desarrollo de cultura educativa en las familias. } \\
\text { - Expandir el capital social de los estudiantes valorizado por las } \\
\text { escuelas. }\end{array}$ \\
\hline $\begin{array}{l}\text { Day y } \\
\text { colaboradores } \\
(2010)\end{array}$ & $\begin{array}{l}\text { Definir sus valores y su visión para aumentar las expectativas, establecer } \\
\text { una dirección y generar confianza. } \\
\text { Remodelar las condiciones para la enseñanza y el aprendizaje. } \\
\text { Reestructurar partes de la organización y rediseñar el liderazgo. } \\
\text { Funciones y responsabilidades. } \\
\text { Enriquecer el plan de estudios. } \\
\text { Mejorar la calidad del docente. } \\
\text { Mejorar la calidad de la enseñanza y el aprendizaje. } \\
\text { Construir colaboración internamente y relaciones fuertes fuera de la } \\
\text { comunidad escolar. }\end{array}$ \\
\hline $\begin{array}{l}\text { Leithwood, Harris } \\
\text { y Hopkins (2008) }\end{array}$ & $\begin{array}{l}\text { El liderazgo escolar es de gran importancia, después de la enseñanza en el } \\
\text { aula, como una influencia en el aprendizaje de los alumnos. } \\
\text { Casi todos los líderes exitosos recurren al mismo repertorio de prácticas } \\
\text { básicas de liderazgo. } \\
\text { Las formas en que los líderes aplican estas prácticas básicas de demuestran } \\
\text { una respuesta a los contextos en los que trabajan, } \\
\text { Los líderes escolares mejoran la enseñanza y el aprendizaje de forma } \\
\text { indirecta a través de su influencia en la motivación, el compromiso y las } \\
\text { condiciones laborales del personal. } \\
\text { El liderazgo escolar tiene una mayor influencia en las escuelas y los } \\
\text { estudiantes cuando se distribuye ampliamente. } \\
\text { Algunos patrones de distribución son más efectivos que otros. } \\
\text { Un pequeño grupo de rasgos personales explica una alta proporción de la } \\
\text { variación en la efectividad del liderazgo. }\end{array}$ \\
\hline
\end{tabular}

Fuente: Elaboración propia.

Una vez seleccionado el estudio de caso y los participantes, nos disponemos a realizar las entrevistas a los diferentes agentes de la comunidad educativa, para ello utilizamos los guiones de entrevista facilitados por ISSPP. En el cuadro siguiente podemos observar resumidamente el número de agentes participantes (un total de 14) así como el número de entrevistas realizadas (14 en total) (cuadro 2). 
Para hacer una investigación más completa y corroborar la información obtenida de las entrevistas se hizo una revisión de documentos del centro, estos fueron: el Proyecto Educativo y el Informe de resultados académicos del curso escolar 2013/2014 que muestran los indicadores homologados para la autoevaluación de centros que imparten Educación Secundaria (AGAVE, 2014). Una vez reunida toda esta información podemos hacer frente al objetivo que se persigue.

Cuadro 2. Número de entrevistas realizadas por agente

\begin{tabular}{lc}
\hline AGENTES PARTICIPANTES & $\begin{array}{c}\text { NÚMERO DE } \\
\text { ENTREVISTAS }\end{array}$ \\
\hline Director & 3 \\
Profesores (3) & 9 \\
Padres y Madres de la AMPA & 1 \\
Alumnado de ESO (seleccionados como representativos) & 1 \\
Total & 14 \\
\hline Fuente: Elaboración propia. &
\end{tabular}

Fuente: Elaboración propia.

Para proceder al análisis de la información, hemos utilizado el análisis de contenido que según Bardín (2002) se resumen en las siguientes fases:

- Preanálisis: análisis del material a través de las lecturas.

- Aprovechamiento del material: reconocimiento de las categorías ofrecidas por el proyecto ISSPP (cuadro 3).

- Tratamiento de resultados, inferencia e interpretación: uso del programa Nvivo 10 para la clasificación de la información y la facilitación de las inferencias e interpretación de los datos recogidos.

\section{Resultados}

\subsection{Describiendo el escenario del centro escolar}

El Instituto de Educación Secundaria en sus comienzos sólo ofertaba Bachillerato, pero a lo largo de los años ha ido creciendo su oferta educativa que consta de Educación Secundaria Obligatoria, Bachillerato, Formación Profesional Básica de diversas ramas y Ciclos Formativos de grado medio y superior, además de ser un centro bilingüe de segunda línea. Para atender esta oferta educativa trabajan 58 profesores, ya que es un centro grande que cuenta un total 595 alumnos.

El contexto en el que se ubica tiene un nivel socio-económico y cultural predominantemente de clase baja, donde hay un alto porcentaje de familias de etnia gitana, la mayoría de los padres tienen un nivel educativo y cultural medio-bajo, contando con la inestabilidad laboral de las familias y la desestructuración de algunas de ellas.

Para atender a toda la diversidad del alumnado, la escuela cuenta con todos los recursos y medios necesarios, con una amplia gama de actividades extraescolares, todas gratuitas. No obstante, consideran que en el centro hay que hacer algunas reformas en instalaciones y material sobre todo informático, estando tales mejoras entre los objetivos del equipo directivo. 
Cuadro 3. Relación de categorías, subcategorías y códigos establecidos

\begin{tabular}{|c|c|c|}
\hline CATEGORIAS & SUBCATEGORÍAS & DEFINICIÓN \\
\hline \multirow{7}{*}{$\begin{array}{l}\text { Centro escolar } \\
\text { (cen. Esc.) }\end{array}$} & Historia del centro (historia) & $\begin{array}{l}\text { Historia del centro desde que comenzó } \\
\text { el director actual }\end{array}$ \\
\hline & Contexto (contex.) & $\begin{array}{l}\text { Características del contexto en el que } \\
\text { está ubicado }\end{array}$ \\
\hline & Desempeño escolar (desempeño) & $\begin{array}{l}\text { Evolución y resultados escolares de los } \\
\text { últimos } 5 \text { años }\end{array}$ \\
\hline & Fortalezas del centro (fortalezas) & Efectos positivos del centro \\
\hline & $\begin{array}{l}\text { Estructura de la gestión del } \\
\text { centro (gestión) }\end{array}$ & $\begin{array}{l}\text { Descripción de la estructura del equipo } \\
\text { directivo del centro }\end{array}$ \\
\hline & $\begin{array}{l}\text { Estructura del currículo } \\
\text { (curriculo) }\end{array}$ & $\begin{array}{l}\text { Descripción del diseño y estructura del } \\
\text { currículo }\end{array}$ \\
\hline & $\begin{array}{l}\text { Implicación de la comunidad } \\
\text { educativa (comunidad) }\end{array}$ & $\begin{array}{l}\text { Nivel de implicación de la comunidad } \\
\text { educativa en el centro }\end{array}$ \\
\hline \multirow{5}{*}{$\begin{array}{l}\text { Biografía del } \\
\text { director } \\
\text { (biogrf.) }\end{array}$} & Historia del director (his. dir.) & Biografía del director del centro \\
\hline & $\begin{array}{l}\text { Satisfacción del trabajo realizado } \\
\text { (satisfacción) }\end{array}$ & $\begin{array}{l}\text { Nivel de satisfacción del trabajo } \\
\text { realizado en el centro }\end{array}$ \\
\hline & Nivel de logro (logro) & $\begin{array}{l}\text { Logros obtenidos por el director en el } \\
\text { centro }\end{array}$ \\
\hline & $\begin{array}{l}\text { Percepciones de liderazgo } \\
\text { (percep.) }\end{array}$ & $\begin{array}{l}\text { Descripción de su percepción sobre el } \\
\text { liderazgo }\end{array}$ \\
\hline & Estrategias de liderazgo (estrat.) & $\begin{array}{l}\text { Descripción de las estrategia/as de } \\
\text { liderazgo llevas a cabo }\end{array}$ \\
\hline \multirow{3}{*}{$\begin{array}{c}\text { Características } \\
\text { y estrategias } \\
\text { de liderazgo } \\
\text { (caract. lider) }\end{array}$} & Desafíos del centro (desafios) & $\begin{array}{l}\text { Objetivos y finalidad que quiere } \\
\text { conseguir el director en el centro }\end{array}$ \\
\hline & $\begin{array}{l}\text { Cualidades del equipo directivo } \\
\text { (equip. dir.) }\end{array}$ & $\begin{array}{l}\text { Describir las cualidades del equipo } \\
\text { directivo }\end{array}$ \\
\hline & $\begin{array}{l}\text { Trabajo con el alumnado } \\
\text { (alumn.) }\end{array}$ & $\begin{array}{l}\text { Descripción de trabajo realizado con el } \\
\text { alumno }\end{array}$ \\
\hline \multirow{5}{*}{$\begin{array}{l}\text { Efectos sobre } \\
\text { la cultura del } \\
\text { centro } \\
\text { (efectos) }\end{array}$} & $\begin{array}{l}\text { Cambios desde la llegada del } \\
\text { director (cambios) }\end{array}$ & $\begin{array}{l}\text { Cambios producidos desde que el } \\
\text { director está al frente en el centro }\end{array}$ \\
\hline & $\begin{array}{l}\text { Efectos sobre la cultura del } \\
\text { centro (efec. cult.) }\end{array}$ & Como ha influido la cultura en el centro \\
\hline & $\begin{array}{l}\text { Efectos sobre el comportamiento } \\
\text { del alumnado (efec. alumn.) }\end{array}$ & $\begin{array}{l}\text { Como ha influido la cultura en el } \\
\text { alumnado }\end{array}$ \\
\hline & $\begin{array}{l}\text { Efectos sobre la comunidad } \\
\text { (efect. comun.) }\end{array}$ & $\begin{array}{l}\text { Como ha influido la cultura en la } \\
\text { comunidad }\end{array}$ \\
\hline & $\begin{array}{l}\text { Aprendizaje sobre el liderazgo } \\
\text { (aprend. lid.) }\end{array}$ & $\begin{array}{l}\text { Aprendizaje obtenido sobre el liderazgo } \\
\text { realizado }\end{array}$ \\
\hline
\end{tabular}

Fuente: Elaboración propia.

El uso de diferentes instrumentos de investigación nos permite justificar la fiabilidad y validez de la investigación, así como la triangulación de la misma (Cohen y Manión, 1990).

En cuanto al análisis de documentos, pudimos corroborar que han mejorado paulatinamente los resultados escolares del alumnado con respecto a años anteriores, y que se constatan con los datos obtenidos en las entrevistas.

En relación al desempeño escolar de este centro, podemos señalar que es elevado puesto que las tasas de éxito escolar son de $90 \%$ del alumnado. Han mejorado considerablemente en los últimos años, sobre todo en Educación Secundaria, desde que se empezó a llevar a 
cabo el proyecto educativo dirigido por el actual equipo directivo, como se refleja en la siguiente referencia:

Cuando empezamos este proyecto había un 50\% de graduados, ahora estamos cerca del 90\%. En Bachillerato estamos en el 80, ahi estábamos en el 60\%. En ciclos formativos también entre el 75 y el 85\% (...) Ese ha sido éxito del proyecto y de los programas que llevamos a cabo. (Director)

No obstante, los profesores y el director señalan que aspiran a que el $100 \%$ del alumnado obtenga el Graduado en Educación Secundaria. Son conscientes de que existe un porcentaje de alumnos que tienen problemas disruptivos, pre-delictivos o graves problemas familiares, lo que lleva a que sea complicado el que consigan el graduado. Su objetivo con este tipo de alumnado es que primero no delincan y adquieran valores y buenos hábitos sociales, como refleja textualmente el director:

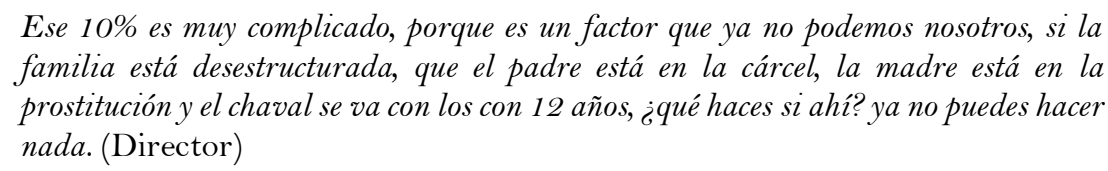

Ese $10 \%$ es muy complicado, porque es un factor que ya no podemos nosotros, si la familia está desestructurada, que el padre está en la cárcel, la madre está en la prostitución y el chaval se va con los con 12 años, ¿qué haces si ahí? ya no puedes hacer nada. (Director)

El objetivo de que todos se gradúen en la ESO es prácticamente es real, pero el problema está en intentar que el alumnado continúe con éxito sus estudios postobligatorios. Es así puesto que al tener que proporcionarle una educación personalizada, según las características de cada alumno, es complicado que en niveles superiores se adapten a las exigencias del currículo y consigan superarlas. De modo, que muchos alumnos se desmotivan y abandonan bien por esa situación o porque deciden matricularse en Bachillerato como segunda opción, puesto que su primera preferencia en muchas ocasiones es realizar un Ciclo de Grado Medio, y lo hacen con una previa desmotivación.

Estas situaciones se ven reflejadas en los porcentajes de éxito escolar del centro, igual que influye considerablemente el número de alumnos absentistas. No obstante, tales porcentajes se han visto reducidos desde que empezó la nueva directiva, aunque sigue siendo una problemática vigente.

Es de resaltar que el equipo directivo estimula y orientan al alumnado para que continúe los estudios obligatorios y postobligatorios, sobre todo a los estudiantes de etnia gitana, que son quienes con más frecuencia abandonan prematuramente el sistema educativo.

\section{Características del centro escolar}

El centro en la actualidad está en una edad de oro, como indican el director y los profesores, no sólo porque va hacer 50 años desde su inauguración, sino porque el centro cuenta con una alta recomendación social tanto que, muy a pesar del equipo directivo, tiene en espera de matrícula a alumnos que no pueden acceder al mismo por estar cubierta la ratio. Todo ello debido a la mejora escolar que se ha producido desde la llegada del director al centro.

El objetivo principal para la dirección es ofrecer una educación de calidad atendiendo a la diversidad mediante el proyecto educativo. Considera que el centro está en una buena situación donde ha visto alcanzado su objetivo, como indica: "He visto hecho realidad el modelo de escuela que deseaba" (Director). Por tanto, su pretensión para los próximos años, es lograr pequeñas metas relativas a reformas del centro y ampliación de la oferta educativa, como se muestra en la cita: 
El salón de actos y el laboratorio de Biología y Geología que sean del siglo XXI. Ese taller de cocina para que aprendan unidades didácticas sobre todos los gitanillos; el Ciclo Formativo de Grado Superior de Actividades Físicas y Deportivas ; $\Upsilon$ ya está!

(Director)

El director apunta que una de las mayores fortalezas que tiene el centro es el trabajo colaborativo entre el profesorado y el equipo directivo para integrar el modelo de escuela por el que apuesta. Es la causa por la que la mayoría de los alumnos, indiferentemente de la diversidad y del nivel educativo en el que se encuentren, consigan obtener el Graduado en la ESO y además continúen, muchos de ellos, sus estudios en la educación postobligatoria.

Esta situación es la gran fortaleza del centro, el que todos apoyen el proyecto educativo, trabajo que fomenta el equipo directivo puesto que aprovecha las virtudes de los profesores, implicándolos y promoviendo su liderazgo para el desarrollo de la mejora del centro. Además, cuenta con un ambiente de confianza entre los profesores, padres, alumnos y la dirección formidable, siendo este un aspecto que valoran sobre todo los profesores.

\section{Estructura de gestión del centro y del currículo}

El equipo directivo fue elegido por el actual director. Como él indica son personas de gran confianza que comparten la misma visión de centro, y que sobre todo apoyan el modelo de escuela que se está desarrollando.

En cuanto a la estructura del currículo, está marcado por la legislación educativa y se someten a ella. No obstante, es de resaltar, que hacen especial incidencia en las adaptaciones curriculares según las necesidades de los estudiantes. Para mejorar su currículo y la calidad de la enseñanza, están empezando a diseñar unidades didácticas integradas, es decir, unidades didácticas donde colaboran todos los departamentos, es lo que llaman el proyecto curricular integrado.

Además, el currículo es complementado, por un lado, con múltiples actividades extraescolares de diversa tipología siendo todas ellas gratuitas (deportivas, lúdicas, talleres de pintura, comic, teatro, etc.). También organizan salidas extraescolares a diferentes lugares de interés para el alumnado e intercambios internacionales a diversos países. A todo ello hay que unir lo que se llama Plan de Acompañamiento para los alumnos que lo necesitan. Consiste en clases de apoyo durante dos días en semana por la tarde. Por otro lado, tiene una amplia selección de planes y proyectos permanentes y transitorios, que van desarrollando para alcanzar la mejora escolar del centro y la convivencia, tan importante para este equipo directivo.

Implicación de la escuela hacia la comunidad y de la comunidad hacia la escuela

Este centro se trabaja arduamente para que haya una buena relación escuela-familias porque lo consideran vital para la mejora escolar. Cuenta con la Asociación de Padres (AMPA) y también con una nueva figura llamada Delegados de Padres que representan a un grupo de clase y que media entre los temas escolares y familiares.

Los estudiantes tienen voz en este centro, pues a través de la figura del Delegado de Clase comentan sus problemáticas y propuestas. Igualmente lo hacen mediante los Alumnos Mediadores de Conflictos que intervienen en cualquier problema que se produce en el centro. 
A parte, trabajan con múltiples asociaciones, con los Servicios Sociales y con el Ayuntamiento de la localidad para mediar entre los conflictos que puedan surgir en la escuela o para realizar actividades de diversa índole. Además, colabora a nivel de intercentro con otras escuelas de la localidad y con la Universidad participando en diversos proyectos, jornadas y actividades.

\subsection{Biografía del director y estilo de liderazgo}

El director del centro tiene una experiencia laboral de treinta y un años habiendo trabajado siempre como orientador. En cuanto a su formación académica es amplia, pues es Diplomado en Audición y Lenguaje y en Ciencias Sociales y Humanas, licenciado en Psicología y doctor en esta misma rama. También ha colaborado durante quince años con la Administración Educativa en proyectos de innovación e impartiendo cursos relacionados con la mejora de la convivencia y de la atención a la diversidad. Y es coautor de un libro y de varios artículos sobre la misma temática.

En relación a su bagaje como líder, nunca antes había ejercido de director, pero ha sido miembro de diferentes equipos directivos como Secretario y Jefe de Estudios. Además, tiene experiencia en la coordinación de diferentes planes, proyectos y programas autorizados por la Administración Educativa durante varios años.

Tras ese bagaje llegó a director en 2010 tras presentar su currículo y el proyecto de dirección. Fue sometido a un proceso de evaluación por parte de la Administración Educativa, y supervisado por la Inspección de Educación de la zona. Fue seleccionado por la vía democrática, el Consejo Escolar de Centro dio el visto bueno y posteriormente hizo los cursos obligatorios para acceder al puesto.

Tan amplia formación y experiencia la ha utilizado en su actual puesto como director para desarrollar el estilo de liderazgo que ejerce.

\section{Satisfacción y motivación con el trabajo realizado}

El director se encuentra muy satisfecho con el modelo de escuela que está llevando acabo, puesto que ha visto hecho realidad, en un contexto tan complicado, el tipo de escuela por el que él apuesta. Considera que es un modelo inclusivo-compensador donde se atiende desde la equidad a la diversidad. Además, cuentan con el apoyo de los profesores y de los padres, quienes confían en el centro y en la formación que se les proporciona a los estudiantes. No obstante, siguen trabajando para conseguir más mejoras escolares y de convivencia.

\section{Nivel de logro ante los retos que plantea en el centro escolar}

El director considera que el logro de los objetivos marcados ha sido lento, pero se han ido consiguiendo, en palabras textuales: "firmaba porque siga así, no es que ya mejore, sino que siga así, es que ya hemos llegado a unos niveles..." (Director).

Los padres confían en la escuela y en el trabajo que están realizando, y las evidencia se encuentra en la satisfacción de sus hijos, los mismos alumnos dicen: "buen ambiente, profesores atentos y el edificio está muy bien” (Alumno 1); “(...) de estar tanto tiempo aquí te acostumbras como en tu casa, te sientes bien aquí” (Alumno 2).

\section{Percepciones del director sobre el rol de liderazgo}

Tiene una percepción del liderazgo participativo, colaborativo y democrático, como el mismo expresa: "el director tiene que estar siempre al servicio de la comunidad educativa 
a la que representa" (Director). Admite que el director tiene que saber dirigir el proyecto hacia la finalidad que se pretende conseguir, pero nunca ser autoritario, dogmático, ni burócrata.

Ejerce un liderazgo en el que deja en un segundo plano la burocracia para centrarse en lo fundamental para él: los alumnos, los padres y los profesores. Piensa que ellos son la verdadera comunidad, apoyándose después en la Administración Educativa, en las Instituciones, etc. y teniendo una buena relación con éstos, como muestra esta cita:

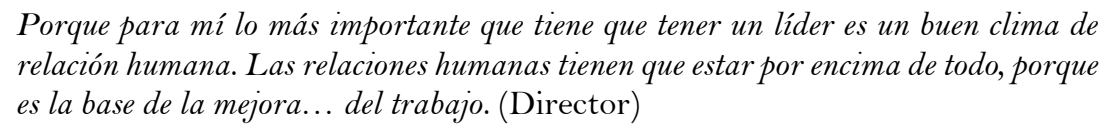

Entre sus roles como director, uno de los más importantes es el de hacer que el profesorado, el alumnado y los padres se sientan útiles obteniendo lo mejor de cada uno, como se muestra textualmente:

\begin{abstract}
Si eso se consigue, pues realmente se hace un centro diferente a los demás. $\Upsilon$ mi empeño ha sido siempre eso". $Y$ se consigue llevando a cabo muchos planes donde se atiende a la diversidad del profesorado, a través de las asociaciones y de las AMPAs y con los alumnos que más lo necesitan. Exponer todos nuestros recursos, ahi es donde más hay que incidir. $Y$ en esa linea se desarrolla el proyecto. (Director)
\end{abstract}

Entre sus funciones principales diarias se encuentra la relación con las familias, puesto que para él es un pilar fundamental. Tienen establecido un sistema de contacto familiar que consiste en realizar compromisos educativos, curriculares y de convivencia. Mensualmente se reúnen para ver la evolución del hijo en el tema concreto a tratar, como se refleja esta cita:

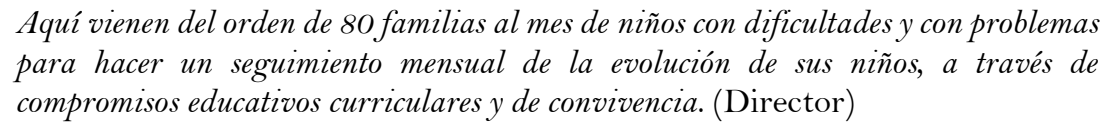

Además de las reuniones trimestrales con los delegados de padres de cada curso.

Igualmente se preocupa de atender a la diversidad del profesorado mediante las reuniones con: los coordinadores de todos los planes y programas que desarrollan en el centro, los coordinadores de áreas de competencias, los equipos educativos, de orientación y de dirección. Luego la atención al alumnado la realiza mediante tareas de orientación académica, profesional y de diagnóstico, pues además de ser director también es orientador.

Entre sus prioridades como líder está la mejora del funcionamiento del centro, y a ello le dedica gran parte de su tiempo. Es fundamental para él conseguir que toda la organización de la escuela esté planificada trimestralmente para que, tanto el equipo directivo como el profesorado, los alumnos y los padres, conozcan en cada momento qué se va hacer, como refleja esta cita: "un centro educativo es algo vivo. Y lo importante es que se optimice el tiempo. La organización del centro tiene que estar prevista” (Director). Apunta que de este modo se evita los malos entendidos y posibles problemas. Podemos concluir este apartado destacando que el eje de sus prioridades como él resalta "son las personas, no son las cosas, no son los papeles (...)" (Director).

\title{
4.3. Características y estrategias del liderazgo
}

Principales desafíos del centro escolar y estrategias exitosas para abordarlos 
La escuela fomenta el compromiso con la comunidad, empezando por la participación de las familias en el centro, según el director hay una alta participación de éstas, aunque siempre sería mejorable. Reconocen que las responsabilidades de las familias llevan a que olviden sus obligaciones con la escuela. En palabras de un profesor:

\begin{abstract}
La sociedad es muy difícil y en España no se potencia, como en otros países, que un padre pueda decir: 'yo tengo una reunión con mi hijo', aquí puede que se rían de ti en tu trabajo. Primero es tu trabajo y luego tu hijo. Pero la implicación de los padres yo creo que es buena, pero a todos nos gustaría que pudieran venir más. (Profesor 1)
\end{abstract}

En relación al éxito escolar y rendimiento del alumno los profesores señalan algunas problemáticas que sufre el centro y con las que tienen que trabajar. En un primer lugar, encontramos el desafío que tiene la escuela para reducir el absentismo y el abandono escolar. Desde que empezó esta nueva directiva los porcentajes se han reducido, pero la problemática persiste sobre todo en los alumnos de etnia gitana y, concretamente, en el género femenino. Los profesores apuntan que intentan retener a algunos alumnos haciendo todo lo que está en su mano y hablando con la familia, pero en muchas ocasiones no es posible.

Por mis manos han pasado tres niñas gitanas que han abandonado a los 15 años y no habido forma humana, y la familia más o menos... Una de las madres pues si era partidaria de que la niña asistiera con regularidad, que faltaba un montón y tal, pero no, ni la madre ni yo pudimos. (Profesor 3)

Además, el abandono y el absentismo no sólo se producen en Educación Secundaria, sino también en Bachillerato. Considera que se debe a que los alumnos en los estudios postobligatorios no reciben una educación adaptada a sus necesidades, como ocurre en la ESO, por lo que en esta etapa les cuesta seguir el nivel que se exige. Eso es lo que les conduce al fracaso escolar y al abandono. Tales situaciones llevan a que se obtenga un porcentaje de alumnado que no asiste a clase y que por lo tanto suspende, y eso hace que las tasas de fracaso escolar aumenten, como señala una profesora:

\title{
Es que ya no tienes la posibilidad de ayudarles ¿̨cómo les ayudas? Si es que no vienen. \\ (...) No es que haya muchísimo, pero sí que hay. (Profesora 2)
}

Finalmente, está la problemática de conducta y disciplina de los alumnos. La convivencia entre alumnos de etnia gitana y los payos en ocasiones se ve agravada, no suele haber demasiados problemas de este tipo, pero sí que sufren algunas situaciones, sobre todo el director y el jefe de estudios que son quienes que se ocupan de resolverlas.

Todos los profesores indican que tales desafíos se han visto disminuidos desde que llegó la nueva dirección. Pues ha llevado a cabo diversas estrategias para intentar resolverlos, como las siguientes:

- Para combatir el absentismo escolar hay una comisión formada por la Jefa de Estudios adjunta, los Servicios Sociales, la Asociación Gitana ROMí y los tutores que tienen esa problemática en su grupo. Hacen reuniones semanales para tratar el tema centrándose en casos concretos de alumnos que faltan a clase con regularidad para tomar soluciones.

- En el caso del absentismo en la enseñanza postobligatoria, se empieza a prevenir en la ESO, con más incidencia en el último curso. Intentan orientar el camino académico de los alumnos y hablar con las familias sobre el fututo del estudiante según sus preferencias. 
- En cuanto a los temas de disciplina el centro cuenta con: el Delegado de Padres, hay un equipo de Alumnos Mediadores de Conflictos que se les forma en la cultura de la mediación para que aprendan a resolver problemas de esta índole, y por último los Delegados de Clase.

En definitiva, en el centro surgen desafíos, pero como el director señala "lo importante no son los problemas, si no el enfoque que se le da, la solución” (Director). Para solucionarlos cuenta con toda la comunidad educativa, y lo hacen desde una perspectiva reeducadora y de servicios a la comunidad.

\section{Cualidades percibidas y contribuciones realizadas por el equipo directivo}

Los profesores indican que el director les transmite su visión de escuela y los objetivos que quiere conseguir igual que lo hace con el resto de su equipo. Consigue que todos visualicen los resultados exitosos que proporciona la implementación del proyecto educativo para lograr que se involucren en el mismo.

Entre las características del director, es de resaltar el que fomente un ambiente de trabajo, de confianza y de colaboración. Es una persona muy responsable de su labor y respetuoso con los demás, apoya el compromiso del profesorado y lo alienta para que continúe sus proyectos y se implique en otros. Está pendiente de todo lo que ocurre en el centro e intenta atender a todos, si está dentro de sus posibilidades. Es un director muy líder y protagonista, tiene totalmente organizado todo tipo de itinerario de los planes y programas, así como las reuniones con los diferentes agentes a lo largo del curso académico. Esa es una de las principales tareas que realiza y una de las claves del buen funcionamiento.

Esta forma de trabajar según los profesores la realiza por el bien común, él tiene una actitud de servicio, como el propio director indica: "nos mueve la mejora de los resultados académicos y del ambiente de convivencia” (Director). Los profesores señalan que le gusta su trabajo, como se muestra en esta referencia: "yo creo que le gusta lo que hace, porque yo nunca he conocido a un director como él, y he estado en un montón de centros" (Profesor 2). Además, señalan que el director no cae en la complacencia y sigue trabajando para mejorar el centro. Es una persona muy comprometida que va a todas las reuniones para conocer la situación de cada alumno, y eso mismo lo corroboran los profesores "sabe todo lo que pasa en el centro y a los alumnos" (Profesor 1).

Por otro lado, los alumnos coinciden en caracterizar al director como una persona atenta, que siempre saca un hueco para atenderlos, muy optimista, que los apoya, es cercano con ellos e intenta conocerlos.

\subsection{Efectos sobre la cultura del centro, el alumnado y la comunidad}

Del cambio de mejora producido con la nueva directiva son conscientes los padres y los alumnos que conocen el centro desde hace más tiempo que incluso alguno de los profesores.

Todos los participantes señalan la buena fama que tiene el centro, destacando la lista de espera que tienen de estudiantes para matricularse en el mismo. Por este motivo, a lo largo del tiempo ha ido ampliando su oferta educativa con los Ciclos de Grado Medio y Superior, la Formación Profesional Básica de diversas áreas y ha cambiado a ser un centro bilingüe. Proporcionando todo ello la posibilidad de ofrecer más opciones a la orientación educativa de los alumnos. 
No solo se ha ofrecido una gran diversidad de atención personalizada y específica a nivel educativo para el alumnado, si no que se ha visto mejorada considerablemente la convivencia en el centro. Mediante los diversos planes y programas, se forma en valores para evitar conflictos y facilitar la convivencia de todos. Es algo que resaltan los diferentes agentes participantes.

Es obvio también para todos los entrevistados, que el director ha ejercido una alta influencia en la cultura del centro. Puesto que las bases del proyecto sobre el que trabaja se centran en la mejora educativa y la convivencia escolar, en base a eso se define su actuación. Podemos empezar señalando la influencia en la cultura escolar entendiendo el cambio de filosofía del centro haciendo saber al profesorado y al resto del equipo directivo el objetivo que persigue. Para ello ha fomentado un ambiente de confianza real y de colaboración entre todos, como resaltan profesores y alumnos: "en este centro se está muy a gusto" (Profesor 1). Por parte de los profesores, lo que más valoran es su liderazgo distribuido y el saber delegar trabajo, además de dirigirlo ofreciendo toda la autonomía en el hacer diario de éstos y de su equipo, aportando el último vistazo de cada acción.

Valoran cómo el director involucra al profesorado en todas las actividades que se realizan en el centro haciéndolos piezas clave en la organización, partiendo de sus principales intereses para que el trabajo realizado sea eficaz. Tal actuación propicia una actitud positiva y de refuerzo en la labor de profesorado, para hacer como él mismo dice: "que se sientan útiles con sus alumnos” (Director).

En todo este proceso los principales actores son los alumnos, y por ello el director piensa que es imprescindible que tengan voz y que no se olviden sus opiniones, porque eso es lo que llevaría a la desconfianza. Además de la cercanía y el trato afectivo que el director tiene hacia ellos, ninguno pasa desapercibido puesto que conoce las situaciones académicas y personales de los mismos.

Como no, la implicación de los padres y madres y de la comunidad en el desarrollo del proyecto para el director es imprescindible, por ello ha conseguido que haya una profunda colaboración de éstos en la escuela.

Tan amplia participación de los actores educativos y la transformación del centro, es debida a la influencia del tipo de liderazgo ejercido por el director y su carisma particular. No debemos olvidar que cuenta con una amplia formación y experiencia que le ha permitido desarrollar este estilo de liderazgo educativo y distribuido donde lo importante "son las personas y no las cosas" (Director) con él señala. Resaltando su actitud de servicio a la comunidad, puesto que ese es su trabajo como personal funcionario del ámbito educativo y como tal así trabaja.

\section{Discusión y conclusiones}

Los resultados de esta investigación muestran que el director desarrolla un liderazgo exitoso que promueve la capacidad de mejora de la escuela. Para ello en primer lugar, tiene unos rasgos y características típicas de este tipo de líder como son: ser una persona muy comprometida con su labor en la escuela, transmitir energía y promover la motivación a la comunidad educativa, aceptar la diversidad y valorarla, transmitir confianza, siente que su trabajo debe estar al servicio de la sociedad y es una persona optimista, empática, carismática y democrática (Day, 2015). 
En segundo lugar, este director realiza estrategias y acciones relacionadas con un liderazgo exitoso como las siguientes:

- El alumnado es el centro de su trabajo en la escuela (Hopkins, 2013).

- Estimula positivamente al alumnado y tiene elevadas expectativas (Day, 2015).

- Estimula el liderazgo del profesorado (Leithwood y Riehl, 2009).

- Trabaja para mejorar el currículo centrándolo en la diversidad y la equidad (Day et al., 2010; Murillo y Hernández-Castilla, 2014).

- Promueve el trabajo colaborativo con el profesorado y con su equipo de dirección (Gronn, 2002; Harris, 2009; Leithwood et al., 2006; Spillane, Camburn y Pareja, 2007; Spillane, Halverson y Diamond, 2004).

- Tiene una visión clara hacia donde se dirige la escuela (Leithwood y Riehl, 2009).

- Comparte la visión que pretende conseguir a su equipo y al profesorado y los hace partícipes (Leithwood y Riehl, 2009; Spillane, 2013).

- Mantiene una visión optimista de la capacidad de mejora (Minor-Rogan y Jacobson, 2014).

- Trabajan en equipo para la toma de decisiones (Beauchamp y Parsons, 2014; Wahistrom y Louis, 2008).

- Promueve un clima de confianza y colaboración (Day et al., 2010; Walker, Lee y Bryant, 2015).

- Es consciente de los desafíos de las escuelas y tiene una elevada preocupación para superarlos (Leithwood y Riehl, 2009).

- Crean nuevas figuras para la resolución de problemas, nuevas actuaciones que conlleva la solución de desafíos persistentes en el centro (Day et al., 2010).

- Establece una gran red de colaboración con la comunidad educativa (Day et al., 2010; Pascual et al., 2016).

Tales resultados como podemos comprobar apoyan los que se obtuvieron en el proyecto ISSPP, relativos a que todos los directores exitosos tienen en común estrategias que llevan a la mejora de la escuela (Day, 2015). Entre las mismas destacan: la personalización de la experiencia de aprendizaje, el trabajo colaborativo de los docentes, la retroalimentación de la comunidad educativa para la mejora de la escuela, las elevadas expectativas individuales y colectivas, y el hecho de ser un director transparente y actuar con integridad, generando respeto y confianza.

Es de resaltar en el modelo de liderazgo de este director, la elevada importancia que le proporciona al proyecto educativo, pues en el mismo se refleja el modelo de escuela que ha conseguido. Comparte a través de él su visión de escuela y los objetivos a alcanzar, estando siempre en el centro el alumnado al que le ofrece una educación desde la diversidad y para la equidad. En su modelo de liderazgo es fundamental el trabajo colaborativo y el ambiente de confianza que se genera en el centro entre la comunidad educativa en general, teniendo en cuenta la opinión de todos ellos (alumnado, profesorado, familias, asociaciones, entidades públicas, etc.) y trabajando en equipo. Tales actuaciones son las que le ha llevado a tener una escuela exitosa situada en un contexto desfavorable. 
El presente estudio se sitúa en la línea uno y dos del proyecto ISSPP que consiste en recopilar datos sobre el liderazgo exitoso desde múltiples perspectivas, es decir, directores, subdirectores, gobernadores, padres, estudiantes y profesores (línea 1) y comparar el liderazgo efectivo en diversos contextos, desde pequeñas escuelas primarias hasta grandes escuelas secundarias (línea 2). En relación a las dos primeras líneas, en esta investigación contextualizada en España no entrevistamos al gobernador porque esa figura educativa como tal no existe en nuestro sistema educativo, de modo que nos limitamos al resto de participantes.

Siguiendo con los niveles del estudio y con las adaptaciones necesarias al contex to español, el mismo abre las puertas para continuar investigando en la línea 3 que se plantea en relación al objetivo de identificar la identidad profesional de los líderes escolares efectivos.

\section{Referencias}

AGAVE. (2014). Indicadores homologados para la autoevaluación de centros que imparten educación secundaria. Sevilla: Consejería de Educación, Cultura y Deporte.

Bardín, L. (2002). Análisis de contenido. Madrid: Akal.

Beauchamp, L. y Parsons, J. (2014). Liderazgo instructivo en Alberta: Hallazgos de la investigación en cinco escuelas altamente eficaces. REICE. Revista Iberoamericana sobre Calidad, Eficacia y Cambio en Educación, 12(4e), 89-98.

Bolívar, A. (2010). Liderazgo para el aprendizaje. Organización y Gestión Educativa. Revista del Fórum Europeo de Administradores de la Educación, 7(1), 1-6.

Bolívar, A. (2014). Building school capacity: Shared leadership and professional learning communities. A research proposal. International Journal of Educational Leadership and Management, 2(2), 147-175.

Cohen, L. y Manión, L. (1990). Métodos de investigación educativa. Madrid: La Muralla.

Day, C. (2015). International successful school principals project (ISSPP): Multi-perspective research on school principals. Nottingham: University of Nottingham.

Day, C. Harris, A. Hadfield M. Tolley, H. y Beresford, J. (2000). Leading schools in times of change. Milton Keynes: Open University Press.

Day, C., Sammons, P., Hopkins, D., Harris, A., Leithwood, K., Gu, Q. y Brown, E. (2010). 10 strong claims about successful school leadership. Nottingham: National College for Teaching and Leadership.

Day, C., Sammons, P., Leithwood, K., Hopkins, D., Gu, Q., Brown, E. y Ahtaridou, E. (2011). Successful school leadership: Linking with learning and achievement. Maidenhead: McGraw Hill Open University Press.

Gronn, P. (2002). Distributed leadership. En K. Leithwood y P. Halliger (Eds.). Second international handbook of educational leadership and administration (pp. 653-696). Dordrecht: Kluwer Academic Publishers. https://doi.org/10.1007/978-94-010-0375-9_23

Hallinger, P. y Heck R. H. (2014). Liderazgo colaborativo y mejora escolar: Comprendiendo el impacto sobre la capacidad de la escuela y el aprendizaje de los estudiantes. REICE. Revista Iberoamericana sobre Calidad, Eficacia y Cambio en Educación, 12(4e), 71-88.

Harris, A. (2009). Distributed leadership: What we know? En A. Harris (Ed.), Distributed leaders. Different perspectives (pp. 11-25). Londres: Springer. https://doi.org/10.1007/978-1-40209737-9 
Hopkins, D. (2013). Exploding the myths of school reform. Victoria: Centre for Strategic Education.

Hopkins, D., Ainscow, M. y West, M. (1994). School improvement in an era of change. Londres: EPPI.

Leithwood, K. y Riehl, C. (2009) ¿Qué sabemos sobre el liderazgo educativo? En K. Leithwood (Coord.), ¿Cómo liderar nuestras escuelas? Aportes desde la investigación (pp.17-34). Santiago de Chile: Área de Educación Fundación Chile.

Leithwood, K., Day, C., Sammons, P., Harris, A. y Hopkins, D. (2006). Seven strong claims about successful school leadership. Nottingham: National College of School Leadership.

Leithwood, K., Harris, A. y Hopkins, D. (2008) Seven strong claims about successful school $\begin{array}{llll}\text { leadership. School Leadership \& } & \text { Management, } & \text { 28(1), 27-42. }\end{array}$ https://doi.org/10.1080/13632430701800060

Minor-Rogan, Y. y Jacobson, S. (2014). USA-Her own words: Turning around an under performing school. En C. Day y D. Gurr (Eds.), Leading school successfully. Stories from the field (pp. 9-18). Londres: Routledge.

Murillo, F. J. y Hernández-Castilla, R. (2014). Liderando escuelas justas para la justicia social. Revista Internacional de Educación para la Justicia Social (RIEJS), 3(2), 13-32.

Pascual, J., Larraguibel, D., Zenteno, D. y Guarda, F. (2016). Liderazgo escolar en tiempos de crisis. el caso de dos liceos del centro sur de Chile después del 27F. REICE. Revista Iberoamericana sobre Calidad, Eficacia y Cambio en Educación, 14(2), 45-62. https://doi.org/10.15366/reice2016.14.2.003

Spillane, J. (2013). The practice of leading and managing teaching in educational organisations. En OECD-Center for Educational Research and Innovation (Ed.), Leadership for 21 st century learning (pp. 59-82). Barcelona: Fundació Jaume Bofill.

Spillane, J., Camburn, E. y Pareja, A. (2007). Taking distributed perspective to the school principal's workday. Leadership and Policy in Schools, 6, 103-125. https://doi.org/10.1080/15700760601091200

Spillane, J., Halverson, R. y Diamond, J. (2004). Towards a theory of leadership practice: A distributed perspective. Journal of Curriculum Studies, 36, 3-34. https://doi.org/10.1080/0022027032000106726

Stoll, L. y Fink, D. (1998). The cruising school: The unidentified ineffective school. En L. Stoll (Ed.), No quick fixes: Perspectives on schools in difficulty (pp. 189-206). Londres: Falmer Press.

Wahistrom, K. L. y Louis, K. (2008). How teachers experience principal leadership: The roles of professional community, trust, efficacy, and shared responsibility. Educational Administration Quarterly, 44, 458-495. https://doi.org/10.1177/0013161X08321502

Walker, A. D., Lee, M. y Bryant, D. A. (2015). How much of a difference do principals make? An analysis of beetwenschools variation in academic achievement in Hong Kong public secondary schools. School Effectiveness and School Improvement, 25, 602-628. https://doi.org/10.1080/09243453.2013.875044 


\section{Breve CV de las autoras}

\section{Estefanía Martínez-Valdivia}

Profesora Sustituta Interina en la Universidad de Jaén. He sido becaria FPU (Formación al Profesorado Universitario). Miembro del Grupo de Investigación "FORCE" y de la Red RILME. Participo como miembro de equipo en proyectos de investigación Nacionales ( $\mathrm{I}+\mathrm{D}+\mathrm{i}$ Liderazgo pedagógico y desarrollo del centro como comunidad profesional: prácticas de éxito en educación obligatoria), y en proyectos internacionales (ISSPP International Successful School Principal Project y Programa de Apoyo al Sector Educativo de MERCOSUR). He publicado artículos y capítulos de libros relativos a la línea de la didáctica, el fracaso escolar y el liderazgo pedagógico, además he participado en diferentes Congresos Nacionales e Internacionales como ponente. Imparto docencia en los Grados de Educación Infantil y Primaria. ORCID ID: 0000-0001-7088-206X. Email: evaldivi@ujaen.es

\section{Inmaculada García-Martínez}

Diplomada en Magisterio especialidad en Educación Primaria. Licenciada en Psicopedagogía. Máster en Investigación e Innovación en Currículum y Formación. Actualmente, es estudiante de doctorado de la Facultad de Educación, en la Universidad de Granada, con una vinculación contractual FPU (Formación del Profesorado Universitario). Es miembro del equipo de investigación FORCE (Formación del Profesorado Centrado en la Escuela; Ref: HUM-386), de la universidad de Granada y miembro de la red de Investigación sobre Liderazgo y Mejora Educativa (RILME). Áreas de investigación: Liderazgo y Coordinación docente en Educación Secundaria; Organización Educativa. ORCID ID: 0000-0003-2620-5779. Email: igmartinez@ugr.es

\section{María Lina Higueras-Rodríguez}

Licenciada en Pedagogía. Máster en Intervención Psicopedagógica. Actualmente, es estudiante de doctorado de la Facultad de Ciencias de la Educación, en la Universidad de Granada, con una vinculación contractual FPU (Formación del Profesorado Universitario). Es miembro del equipo de Investigación FORCE (Formación del Profesorado Centrado en la Escuela) y miembro de la Red de Investigación sobre Liderazgo y Mejora Educativa (RILME). Las áreas de investigación son: Formación del profesorado, Innovación y Liderazgo. Actualmente trabaja dentro del Proyecto Internacional ISSPP. ORCID ID: 0000-0002-4458-7339. Email: mlina@ugr.es 